\title{
Complex Pattern Formations by Spatial Varying Parameters
}

\author{
Siqing $\mathrm{Li}^{1,2, *}$ and Leevan $\mathrm{Ling}^{2}$ \\ ${ }^{1}$ College of Mathematics, Taiyuan University of Technology, Taiyuan, Shanxi 030024, \\ China \\ 2 Department of Mathematics, Hong Kong Baptist University, Kowloon Tong, \\ Hong Kong
}

Received 25 December 2018; Accepted (in revised version) 6 December 2019

\begin{abstract}
Pattern formations by Gierer-Meinhardt (GM) activator-inhibitor model are considered in this paper. By linear analysis, critical value of bifurcation parameter can be evaluated to ensure Turing instability. Numerical simulations are tested by using second order semi-implicit backward difference methods for time discretization and the meshless Kansa method for spatially discretization. We numerically show the convergence of our algorithm. Pattern transitions in irregular domains are shown. We also provide various parameter settings on some irregular domains for different patterns appeared in nature. To further simulate patterns in reality, we construct different kinds of animal type domains and obtain desired patterns by applying proposed parameter settings.
\end{abstract}

AMS subject classifications: $35 \mathrm{~K} 57,65 \mathrm{M} 70$

Key words: Gierer-Meinhardt model, pattern formation, meshless method, spatially varying parameter.

\section{Introduction}

Many biological process, including animal pigmentation $[8,33,35,36]$, tumor formation [16] and animal population distribution [9], can be modeled by nonlinear reaction diffusion systems. Mathematical analysis and simulation can help researchers better understanding interaction between chemicals in animal growth from a single cell to large amount patterns we see in nature. In 1952, Turing proposed the first model for morphogens phenomenon in his paper [40]. In the paper, he studied mathematical models, which can generate the organism structure from a zygote. Prompted by this study, many Turing models were put forward for different biological and physical process in nature.

*Corresponding author.

Emails: lisq3@sustech.edu.cn (S. Q. Li), lling@hkbu.edu.hk (L. Ling) 
In 1955, a mathematical model of some threshold phenomenon in the nerve membrane was posed by Fitzhugh in [19]. In 1979, Schnakenberg [39] proposed a model to describe trimolecular autocatalytic reactions with two chemicals. The Gierer-Meinhardt activatorinhibitor model was first introduced by Gierer and Meinhardt in [20] to describe the spatial pattern of tissue structures starting from almost homogeneous tissue.

Most Turing models can be written by reaction diffusion systems with two chemicals as

$$
\left\{\begin{array}{l}
\frac{\partial u}{\partial t}=D_{u} \Delta u+f(u, v), \\
\frac{\partial v}{\partial t}=D_{v} \Delta v+g(u, v),
\end{array}\right.
$$

with $u(x, t)$ and $v(x, t)$ denoting concentrations of two chemicals at spatial position $x$ and time $t, D_{u}$ and $D_{v}$ being diffusion constants. Functions $f(u, v)$ and $g(u, v)$ have different representations in different models and they describe the reactions of two chemicals. In this paper, we consider the Gierer-Meinhardt (GM) model [30] with

$$
\left\{\begin{array}{l}
f(u, v)=k_{1}+k_{3} \frac{u^{2}}{v}-k_{2} u, \\
g(u, v)=k_{4} u^{2}-k_{5} v,
\end{array}\right.
$$

for some positive parameters $k_{1}, \cdots, k_{5}$. By the manipulation in [34], the GM model can be written in a non-dimensional form

$$
\left\{\begin{array}{l}
f(u, v)=r\left(1+\frac{u^{2}}{v}\right)-\mu u, \\
g(u, v)=r u^{2}-v v .
\end{array}\right.
$$

where $r, v$ and $\mu$ are positive constants.

Based on some linear analysis, we will study Turing instability of the GiererMeinhardt model for fixed bifurcation parameter. Numerical study for pattern formation is also essential since the non-uniform solution corresponding to spatial patterns cannot be found analytically. For time discretization, numerical schemes can be chosen with different convergence behavior. The Runge-Kutta method used in $[13,38]$ and the Crank-Nicolson scheme was employed in $[22,23]$. The alternating direction implicit Crank-Nicholson (ADI-CN) was applied in [17] to solve two dimensional Riesz space fractional diffusion equations. In [18], the authors proposed a two level method for semilinear reaction-diffusion equations. We use the implicit-explicit SBDF2 scheme [37] in this paper which also be employed in [11]. Different numerical methods are also proposed for the spatial discretization, such as the finite difference method $[8,35,36]$, the finite element method $[28,41]$ and different kinds of meshless methods (the element free Galerkin method [14, 29], the local radial basis function method [38]), ect. Compared to methods with mesh, meshless methods are easier applied to irregular domains, for instance ellipse domains and butterfly shape domains considered in [29]. Kansa method, proposed by E. 
J. Kansa in 1990 [24,25], is a typical meshless method to solve partial differential equations by imposing strong form collocation to PDEs. To overcome the solvability problem of Kansa method formed in [21], overdetermined Kansa method was applied to solve PDEs in [26]. Convergence results of the overdetermined Kansa method were proved in $[12,27]$. Spectral methods are another kind of meshless method which are widely used for solving different problems, for instance, flexible multibody dynamics [31] and for time-fractional advection dispersion equation [32].

In [35], the effects of spatially varying parameters on patterns formation in two dimension domains were explored. Since the finite difference methods was used, only square domains considered in the paper. In our work, we study the Turing instability and pattern formations by spatially varying parameters of the Gierer-Meinhardt model in different irregular domains. By applying the second order semi-implicit backward difference formula (SBDF2) to the time discretization and the Kansa method to the spatially discretization introduced in Section 3, pattern formations as bifurcation parameter changing are studied in Section 4 . Besides constant parameter in spatially space, complex patterns are also formed by changing the bifurcation parameter continuously or discontinuously. To further simulate patterns in reality, we also construct different kinds of animal type domains and construct expected patterns by employing proposed parameter settings on different patches of domains.

\section{Turing instability}

In this section, we study the Turing instability of GM model by linear analysis. The system (1.1) subject to Neumann boundary conditions (zero flux) is considered as:

$$
\frac{\partial u}{\partial n}=0=\frac{\partial v}{\partial n} \quad \text { on } \partial \Omega,
$$

with $n$ being the unit outward normal vector of the boundary. Zero flux boundary conditions imply that the system is self-organization with no external input. The steady state $\left(u_{\infty}, v_{\infty}\right)$ of the system (1.1) with function $f$ and $g$ in Eq. (1.2) is given

$$
u_{\infty}=\frac{r+v}{\mu} \quad \text { and } \quad v_{\infty}=\frac{r(r+v)^{2}}{v \mu^{2}}
$$

which can be obtained by solving $f\left(u_{\infty}, v_{\infty}\right)=0$ and $g\left(u_{\infty}, v_{\infty}\right)=0$. In Eq. (1.2), we set the bifurcation parameter as $\mu$ and treat parameters $v$ and $r$ as constants. When the other parameters are fixed, the Turing instability appears when $\mu$ is larger than some critical value. In the following, we will study conditions for Turing instability by the method in [7].

The Turing instability, also known as diffusion-driven instability, means the situation where the solution is stable in the absence of diffusion, but unstable in the presence of diffusion. Conditions for Turing instability can be analysed by studying the behaviors 
of the system with a small perturbation introduced to the steady state as $\widetilde{u}=u-u_{\infty}$ and $\widetilde{v}=v-v_{\infty}$. Without considering diffusion terms, by the Taylor expansions of functions $f$ and $g$ at $\left(u_{\infty}, v_{\infty}\right)$, we obtain

$$
\left(\begin{array}{c}
\widetilde{u}_{t} \\
\widetilde{v}_{t}
\end{array}\right)=A\left(\begin{array}{l}
\widetilde{u} \\
\widetilde{v}
\end{array}\right) \quad \text { with } A=\left.\left(\begin{array}{ll}
f_{u} & f_{v} \\
g_{u} & g_{v}
\end{array}\right)\right|_{\left(u_{\infty}, v_{\infty}\right)}=\left(\begin{array}{cc}
\frac{\mu(v-r)}{r+v} & -\frac{v^{2} \mu^{2}}{r(v+r)^{2}} \\
\frac{2 r(v+r)}{\mu} & -v
\end{array}\right) \text {, }
$$

where $\widetilde{u}_{t}$ represent the derivative of $\widetilde{u}$ to $t$ and $f_{u}$ is the derivative of function $f$ to $u$. Similar expressions are used for $\widetilde{v}_{t}, f_{v}, g_{u}$ and $g_{v}$. The system is stable if and only if all eigenvalues have negative real part $\left(\operatorname{Re}\left(\lambda_{i}\right)<0, i=1,2\right)$ with $\lambda$ computing by solving $|A-\lambda I|=0$ as

$$
\lambda=\frac{\operatorname{Tr}(A) \pm \sqrt{(\operatorname{Tr}(A)-4|A|)}}{2} .
$$

Therefore, linearly stability is guaranteed by two conditions:

$$
\begin{aligned}
& \operatorname{Tr}(A)=f_{u}+g_{v}=\frac{\mu(v-r)}{r+v}-v<0, \\
& |A|=f_{u} g_{v}-f_{v} g_{u}=-v \frac{\mu(v-r)}{r+v}+\frac{2 v^{2} \mu}{(v+r)}>0 .
\end{aligned}
$$

After reconsidering the diffusion term, the reaction-diffusion system becomes

$$
\left(\begin{array}{l}
\widetilde{u}_{t} \\
\widetilde{v}_{t}
\end{array}\right)=A\left(\begin{array}{l}
\widetilde{u} \\
\widetilde{v}
\end{array}\right)+D \nabla^{2}\left(\begin{array}{l}
\widetilde{u} \\
\widetilde{v}
\end{array}\right) \quad \text { with } D=\left(\begin{array}{cc}
D_{u} & 0 \\
0 & D_{v}
\end{array}\right) \text {, }
$$

whose general solutions is

$$
\widetilde{u}=\sum_{k} E_{k} e^{\lambda(k) t} u_{k}(x),
$$

with $E_{k}$ being the Fourier coefficients of the initial condition and $u_{k}$ being the eigenfunction of $\Delta u_{k}+k^{2} u_{k}=0$ with zero flux boundary condition. For example, in the rectangle domain $\Omega=:[-p, p] \times[-q, q]$, solutions become:

$$
\widetilde{u}=\sum_{m, n} E_{m, n} e^{\lambda_{m, n} t} \cos \left(\frac{m \pi x}{p}\right) \cos \left(\frac{n \pi y}{q}\right) .
$$

The same argument can be used to obtain the solution for $\widetilde{v}$. The eigenvalues can be got by solving following characteristic equation

$$
\lambda^{2}+\left(\left(D_{u}+D_{v}\right) k^{2}-\operatorname{Tr}(A)\right) \lambda+h\left(k^{2}\right)=0,
$$

with $h\left(k^{2}\right)=D_{u} D_{v} k^{4}-\left(D_{u} g_{v}+D_{v} f_{u}\right) k^{2}+|A|$ and pattern formations occur when $\operatorname{Re}\{\lambda(k)\}>0$ for some $k \neq 0$. Combining the conditions in (2.2a) and (2.2b), the eigenvalue with positive real part can be obtained when $h\left(k^{2}\right)<0$. This can be ensured by 
following two conditions

$$
\begin{aligned}
& D_{u} g_{v}+D_{v} f_{u}>0, \\
& \left(D_{u} g_{v}+D_{v} f_{u}\right)^{2}>4 D_{u} D_{v}|A| .
\end{aligned}
$$

The condition in Eq. (2.6b) is to make the minimum value of $h\left(k^{2}\right)$ to be negative.

Combining conditions in Eqs. (2.2a), (2.2b), (2.6a), and (2.6b), we can compute the critical value of $\mu$ in our model is

$$
\mu_{T}=\frac{d v((3 v+r)(v+r)+2(v+r) \sqrt{v(2 v-r)})}{(v-r)^{2}} .
$$

\section{The Meshless methods for pattern formations}

In order to study pattern formations as the bifurcation parameter changes, an iterative numerical scheme is needed. In Eq. (1.1), an second order semi-implicit backward difference formula (SBDF2) [37] is employed to deal with the time as

$$
\left\{\begin{array}{l}
\frac{3 u^{n+1}-4 u^{n}+u^{n-1}}{2 \Delta t}=D_{u} \Delta u^{n+1}+2 f\left(u^{n}, v^{n}\right)-f\left(u^{n-1}, v^{n-1}\right), \\
\frac{3 v^{n+1}-4 v^{n}+v^{n-1}}{2 \Delta t}=D_{v} \Delta v^{n+1}+2 g\left(u^{n}, v^{n}\right)-g\left(u^{n-1}, v^{n-1}\right),
\end{array}\right.
$$

where $\Delta t$ is time step, $u^{n}$ and $v^{n}$ are concentration of $u$ and $v$ at time $t=n \Delta t$. After simplifying, we obtain

$$
\left\{\begin{array}{l}
\frac{3}{2} u^{n+1}-\Delta t D_{u} \Delta u^{n+1}=2 \Delta t f\left(u^{n}, v^{n}\right)-\Delta t f\left(u^{n-1}, v^{n-1}\right)+2 u^{n}-\frac{1}{2} u^{n-1}, \\
\frac{3}{2} v^{n+1}-\Delta t D_{v} \Delta v^{n+1}=2 \Delta t g\left(u^{n}, v^{n}\right)-\Delta t g\left(u^{n-1}, v^{n-1}\right)+2 v^{n}-\frac{1}{2} v^{n-1} .
\end{array}\right.
$$

From Eq. (3.1), concentration of $u$ and $v$ at time $(n+1) \Delta t$ can be evaluated by using their values at time $n \Delta t$ and $(n-1) \Delta t$.

For the spatial discretization, we use the Kansa method. Let $Z=\left\{z_{1}, \cdots, z_{N}\right\}$ be a set of discrete trial centers in domain $\Omega$ and $\Phi(\cdot, \cdot)$ be a radial basis function. The concentration function $u^{n+1}$ can be approximated as

$$
u^{n+1}=\sum_{i=1}^{N} \lambda_{i} \Phi\left(\cdot, z_{i}\right)
$$

with unknown coefficients $\Lambda=\left[\lambda_{1}, \cdots, \lambda_{N}\right]^{T}$. In the domain $\Omega$, from Eq. (3.1), strong form collocation conditions are imposed as

$$
\sum_{i=1}^{N} \lambda_{i}\left(\frac{3}{2} \sum_{j=1}^{N_{i}} \Phi\left(z_{j}, z_{i}\right)-\Delta t D_{u} \sum_{j=1}^{N_{i}} \Delta \Phi\left(z_{j}, z_{i}\right)\right)=h\left(z_{j}\right) \quad \text { for } z_{j} \in \Omega,
$$


where

$$
h\left(z_{j}\right)=2 \Delta t f\left(u_{j}^{n}, v_{j}^{n}\right)-\Delta t f\left(u_{j}^{n-1}, v_{j}^{n-1}\right)+2 u_{j}^{n}-\frac{1}{2} u_{j}^{n-1}
$$

with $u_{j}^{n}$ denotes concentration of $u$ at $z_{j}$ and $t=n \Delta t$. On the boundary, zero flux boundary conditions are imposed as

$$
\frac{\partial u^{n+1}}{\partial n}=\sum_{i=1}^{N} \lambda_{i} \sum_{j=1}^{N_{b}} \frac{\partial \Phi\left(z_{j}, z_{i}\right)}{\partial n}=0 \quad \text { for } z_{j} \in \partial \Omega .
$$

Combined collocation conditions in the domain and on the boundary, unknown coefficients $\Lambda$ for $u^{n+1}$ can be obtained by solving following matrix equation

$$
\left[\begin{array}{c}
\frac{2}{3} \Phi\left(Z_{i}, Z\right)-\Delta t D_{u} \Delta \Phi\left(Z_{i}, Z\right) \\
\frac{\partial \Phi\left(Z_{b}, Z\right)}{\partial n}
\end{array}\right] \Lambda=\left[\begin{array}{c}
h\left(Z_{i}\right) \\
0
\end{array}\right]
$$

with $Z=Z_{i} \cup Z_{b}$. After finding coefficients $\Lambda$, the concentration value $u^{n+1}$ in the domain $\Omega$ is given by Eq. (3.2).

Since the Kansa method in Eq. (3.3) for the spatially discretization is meshless, it can be applied to irregular domains. We will mainly consider irregular domain shapes in our simulation.

\section{Complex pattern formations by the spatial varying parameters}

The Whittle-Matern-Sobolev kernels have the advantage that the stability and convergence of the unsymmetric meshless collocation methods for elliptic PDEs by the kernel had be proved in the papers $[12,27]$. Therefore, for all tested examples, the scaled WhittleMatárn-Sobolev kernel

$$
\Phi_{m}(x)=:\|c x\|_{2}^{m-d / 2} \mathcal{K}_{m-d / 2}\left(\|c x\|_{2}\right) \text { for } x \in \mathbb{R}^{d},
$$

is used where $K_{v}$ is the Bessel functions of the second kind and $c$ is the scale factor. The time step is fixed as $\Delta t=0.01$.

\subsection{Convergence and stability of the numerical method}

In order to show the efficiency of our method to solving the Turing models, instead of GM model, we use a model for convergence test based on Brusselator as in [15, Example 1]. With system equations in Eq. (1.1), the reaction functions $f(u, v)$ and $g(u, v)$ in the Brusselator model have representations

$$
f(u, v)=B+u^{2} v-(A+1) u, \quad g(u, v)=A u-u^{2} v .
$$


The parameters are set as $D_{v}=1, A=1, B=0.5, D_{u}=1$. We solve the problem in the unit square domain $\Omega=[0,1]^{2}$ with the exact solutions as

$$
u^{*}=\cos (t) \cos (2 \pi x) \cos (\pi y), \quad v^{*}=\cos (t) \cos (\pi x) \cos (2 \pi y) .
$$

The initial conditions are generated from the exact solutions as:

$$
u(x, y, 0)=\cos (2 \pi x) \cos (\pi y), \quad v(x, y, 0)=\cos (\pi x) \cos (2 \pi y) .
$$

In order to ensure the exact solutions of the system, instead of the original functions $f(u, v), g(u, v)$, we construct the new reaction diffusion functions $F(u, v), G(u, v)$ as

$$
F(u, v)=\tilde{f}(x, y, t)+f(u, v), \quad G(u, v)=\tilde{g}(x, y, t)+g(u, v),
$$

with

$$
\tilde{f}(x, y, t)=\frac{\partial u^{*}}{\partial t}-D_{u} \Delta u^{*}-f\left(u^{*}, v^{*}\right), \quad \tilde{g}(x, y, t)=\frac{\partial v^{*}}{\partial t}-D_{v} \Delta v^{*}-g\left(u^{*}, v^{*}\right) .
$$

The Neumann boundary conditions are imposed on the boundary. We compute the $L^{2}(\Omega)$ error for numerical solutions for the fixed time $T$ as

$$
e_{h}=\sqrt{e_{h, u}^{2}+e_{h, v}^{2}}
$$

with

$$
e_{h, u}=\left\|u^{n}-u^{*}\right\|_{L^{2}} \approx \frac{1}{N} \sqrt{\sum_{n=1}^{N}\left(u_{i}^{n}-u_{i}^{*}\right)^{2}}, \quad e_{h, v} \approx \frac{1}{N} \sqrt{\sum_{n=1}^{N}\left(v_{i}^{n}-v_{i}^{*}\right)^{2}},
$$

with $N$ being the number of evaluation points in the domain.

With fixed kernel smoothness $m=6$, final time $T=1$ and time step $\Delta t=0.01$, Fig. 1 shows the numerical results under different RBF scaler parameters $c$. It can be seen that small scaler parameter $c=1$ results in the smallest numerical errors. However, it also leads the largest condition number of the collocation matrix in Eq. (3.3) and cannot obtain the right numerical solution for the cases $n_{Z}>250$. For large scale parameter $c=10$, we have the smallest condition number and the largest $L^{2}(\Omega)$ error. Therefore, we use scaler parameter $c=5$ in the following to balance the condition number of the collocation matrix and the accuracy of our scheme.

Next, we consider the convergence behaviors and stability of our method. By fixing $c=5$ and time step $\Delta t=0.01$, Fig. 2(a) shows the convergence of the solution. For fixed kernel smoothness, numerical solution converges to exact solution as the discrete points increase. What's more, higher accuracy and convergence rate can be obatined by using higher kernel smoomthness $m$. Under uniformly distributed discrete set with $n_{Z}=31^{2}$, Fig. 2(b) shows the $L^{2}(\Omega)$ error as time $t$ increase. It can be seen again that higher kernel smoothness leads to higher accuray solutions for fixed $t$. For all tested $m$, the numerical 


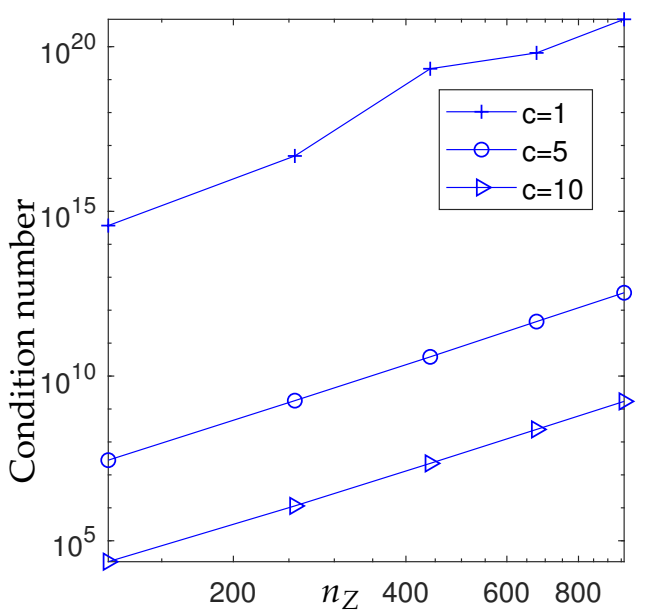

(a)

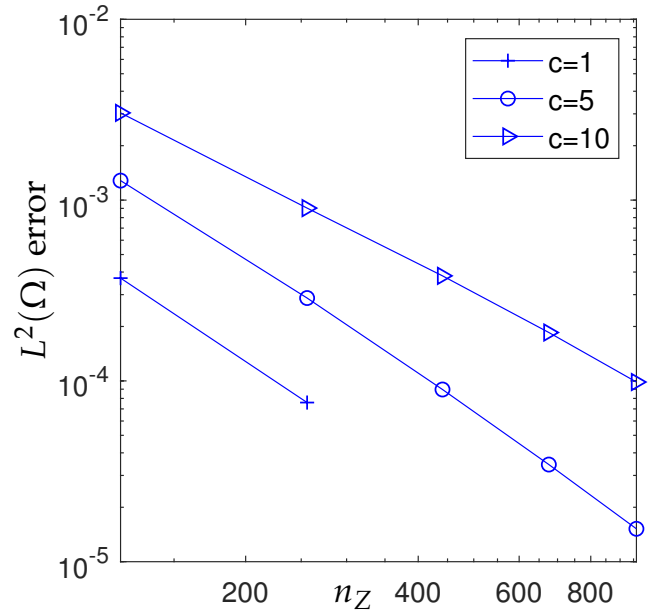

(b)

Figure 1: For the Brusselator model in the unit square domain with zero flux boundary condition, when use kernel smoothness $m=6$, final time $T=1$ and time step $\Delta t=0.01$, under different RBF scaler parameters $c$, (a) the condition number of the collocation matrix; (b) the $L^{2}(\Omega)$ error of the numerical solution.

errors are monotone, which is evidence that our method is stable. To show the robustness of our method, we apply the halton points in the domain and regular points on the bundary. Fig. 2(c) shows the distribution of points for $n_{Z}=21^{2}$ and Fig. 2(d) shows that we can have convergent solutions for different kernel smoothness under the irregularly distributed points.

The model to test the convergence of our sheme in the above was also considered in [15, Example 1]. The ADI extrapolated Crank-Nicolson orthogonal spline collocation method was used to solve the model in [15]. Under same discrete sets, $A, B, D_{u}$ and $D_{v}$, Tables 1 and 2 show the $L^{2}(\Omega)$ error and convergence rate of the solution for $c=5, \Delta t=$ $0.01, T=1$. The convergence rate is computed as

$$
\text { Rate }=\frac{\log \left(e_{h_{1}} / e_{h_{2}}\right)}{\log \left(h_{1} / h_{2}\right)}
$$

In [15, Example 1], the order of convergence rate to discrete sets is $\mathcal{O}\left(h^{r+1}\right)$ in $L^{2}(\Omega)$ error with $r$ being the degree of polynomial used. From Table 1, although the accuracy of our method at $m=6$ is not higher than results in [15, Example 1] at $r=3$, similar convergence rates are obtained. From Table 2, the convergence rates of our method at $m=8$ are almost two order higher than in [15, Example 1] at $r=5$. This means that the accuray of the numerical solutions may same with the method in [15] as discrete points increase. For both cases, we can see that more CPUtime used as the increase of discrete points. 


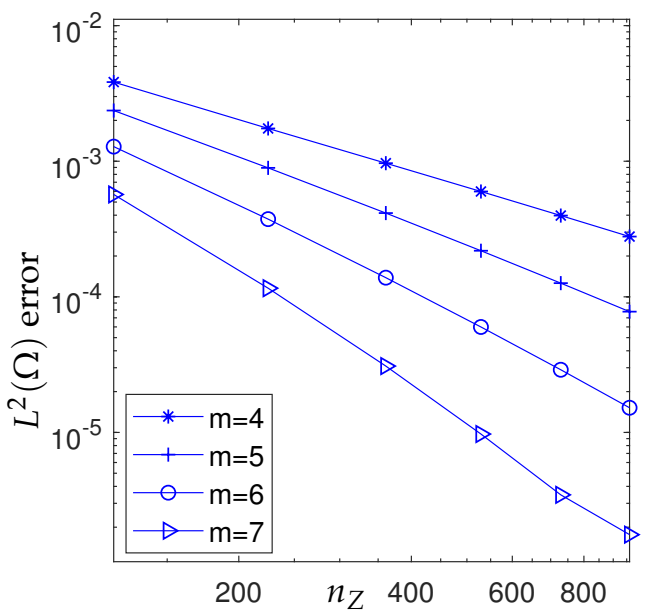

(a)

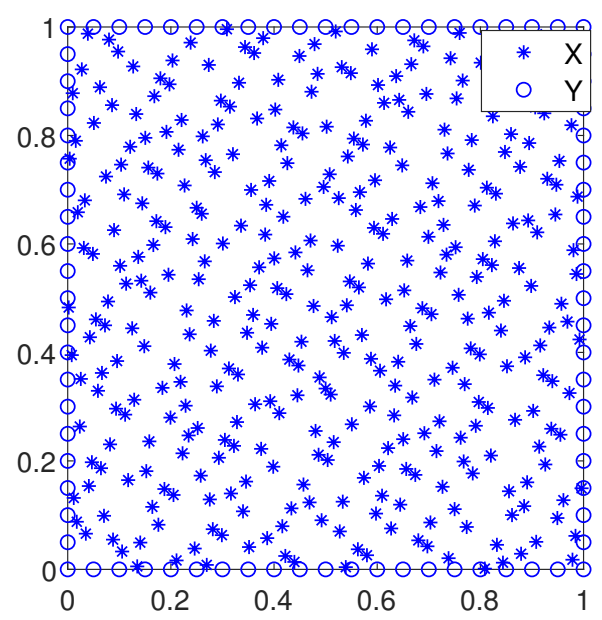

(c)

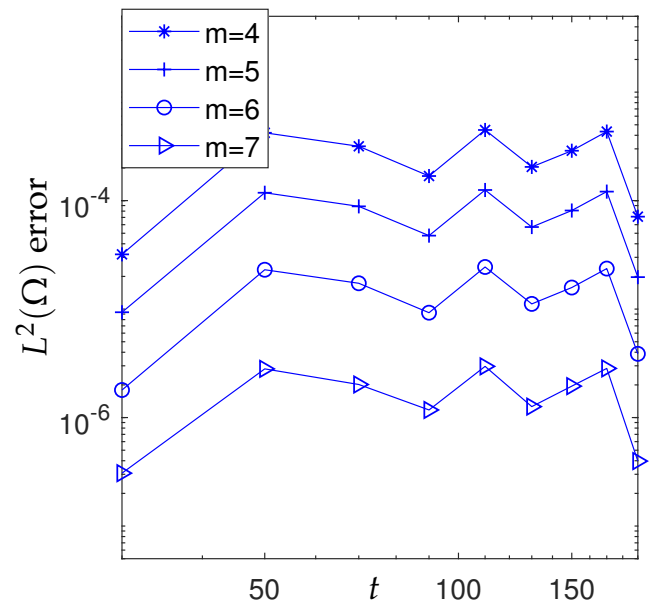

(b)

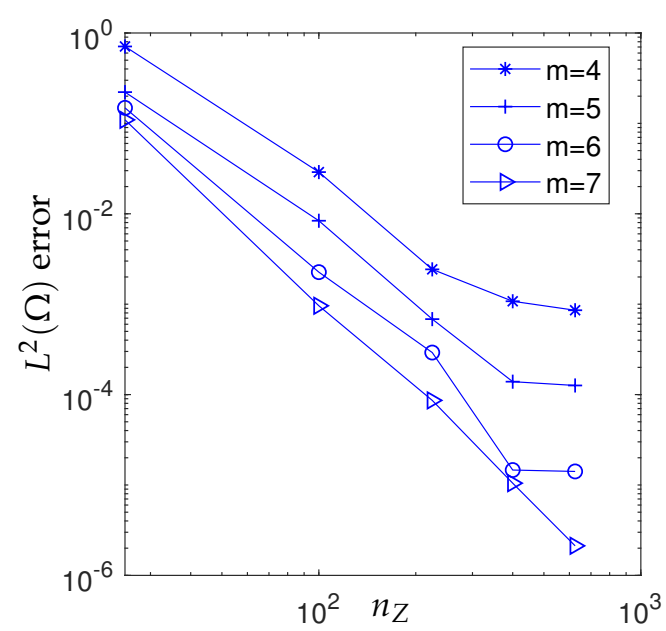

(d)

Figure 2: For the Brusselator model in the unit square domain with zero flux boundary condition, when use RBF scale $c=5$ and time step $\Delta t=0.01$, under different kernel smoothness, (a) the convergence behavior of the numerical solution with final time $T=1$, (b) the $L^{2}(\Omega)$ error as time increase with $n_{Z}=31^{2}$, (c) halton points in the domain and uniformly distributed points on the boundary with $n_{Z}=21^{2}$ (d) convergence behavior of numerical solution by irregular points.

\subsection{Pattern formations for spatially constant bifurication parameter}

In this part, we study patterns for spatially fixed $\mu$. The unit square domain $[0,1] \times[0,1]$ is firstly considered. For the Kansa method, we use $40^{2}$ uniformly distributed points in $\Omega$. when we fix parameter as $D_{v}=0.27, v=100.0, r=0.001, D_{u}=d D_{v}, d=0.0035$, by the Turing instability conditions in $(2.2 a),(2.2 b),(2.6 a)$ and $(2.6 b)$, the critical value of bifurcation parameter $\mu$ to ensure pattern formations is 2.04 . In the system (1.1), the 
Table 1: CPUtime, $L^{2}(\Omega)$ errors and convergence rates comparsion with [15, Example 1] at $\Delta t=0.01, T=1$, $m=6$.

\begin{tabular}{||cccccc||}
\hline$n_{Z}$ & $e_{h, u}$ & CPUtime & Rate & $e_{h_{1}}(r=3[15])$ & Rate \\
\hline $10^{2}$ & $1.20 * 10^{-3}$ & 0.0132 & & $0.6 * 10^{-4}$ & \\
$15^{2}$ & $2.42 * 10^{-4}$ & 0.0509 & 4.01 & $0.13 * 10^{-4}$ & 4.01 \\
$20^{2}$ & $7.16 * 10^{-5}$ & 0.0865 & 4.22 & $0.42 * 10^{-5}$ & 4.00 \\
\hline
\end{tabular}

Table 2: CPUtime, $L^{2}(\Omega)$ errors and convergence rates comparsion with [15, Example 1] at $\Delta t=0.01, T=1$, $m=8$.

\begin{tabular}{||cccccc||}
\hline$n_{Z}$ & $e_{h, u}$ & CPUtime & Rate & $e_{h_{1}}(r=5[15])$ & Rate \\
\hline $10^{2}$ & $2.46 * 10^{-4}$ & 0.0114 & & $0.81 * 10^{-6}$ & \\
$15^{2}$ & $1.02 * 10^{-5}$ & 0.0469 & 7.89 & $0.71 * 10^{-7}$ & 6.00 \\
$20^{2}$ & $9.82 * 10^{-7}$ & 0.0882 & 8.19 & $0.12 * 10^{-7}$ & 6.00 \\
\hline
\end{tabular}

initial conditions are set as

$$
u(0, x)=u_{\infty}+0.01 \xi
$$

with $\xi$ denoting a uniform random number in $[-1,1]$ and $u_{\infty}$ being spatially steady state. We show patterns at $\mu \in\{2.04,2.05,2.15,2.4,3\}$ in Fig. 3 under two different random perturbations in Eq. (4.1) to study sensitivity of patterns to initial conditions. From Figs. 3(a)(d), concentrations of $u$ keep at steady state in domain for $\mu=2.04$ and patterns appear for $\mu>2.04$, which is consistent with Turing instability analysis in Section 2. Numerical experiments show that strips are formed for $\mu \in[2.1,2.3]$. Figs. 3(e) and (f) show stable states for $\mu=2.15$ at final time $T=3000$. When $\mu$ takes any value between 2.3 and 2.5, bistable patterns are formed in which both striped and spotty patterns are possible states. Figs. $3(\mathrm{~g})$ and $(\mathrm{h})$ show results for $\mu=2.4$ at $T=3000$. For $\mu>2.5$, spots are formed with their size becoming smaller as $\mu$ increasing. Figs. 3(i) and (g) are the resulting patterns when $\mu=3$. For any fixed value $\mu$, the same type but different patterns are formed by Kansa method under different initial conditions from Fig. 3, for instance, the direction of strips for $\mu=2.15$, the position of spots and strips for $\mu=2.4$, locations of spots for $\mu=3$.

From [10], pattern formations are sensitive to domain shapes for some Turing systems. Since we aim to simulate complicated patterns on irregular domains, it is vital to investigate effect of domain shapes to pattern transition in the GM model. Specifically, we consider the peanut shape domain $\Omega_{1}$ defined in Eq. (A.1) with similar curve on fish and the asterisk shape domain $\Omega_{2}$ defined in Eq. (A.2) with its boundary similar to octopus. For the domain $\Omega_{1}$, we use $N_{i}=884$ discrete points in the domain and $N_{b}=222$ equally spaced discrete points on the boundary. Uniformly distributed $N_{i}=969$ and $N_{b}=260$ points are used in the domain $\Omega_{2}$ and on the boundary respectively. Fig. 4 shows pattern formations for $\mu \in\{2.04,2.05,2.15,2.4,3\}$ of these two domains. We can see almost the same behavior as what happened in the square domain. Therefore, domain shapes do not have detectable influence on pattern transitions in the GM model. 


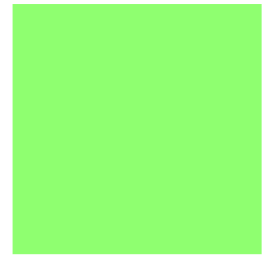

(a) $\mu=2.04, T=400$

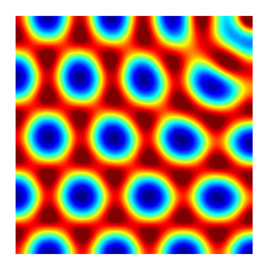

(c) $\mu=2.05, T=3000$

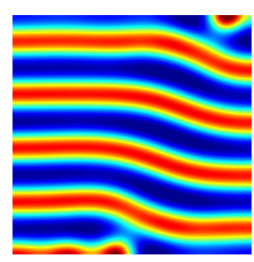

(e) $\mu=2.15, T=3000$

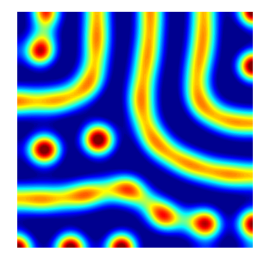

(g) $\mu=2.4, T=1000$

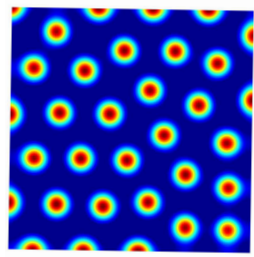

(i) $\mu=3, T=400$

Kansa method 1

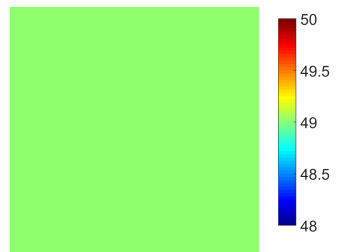

(b) $\mu=2.04, T=400$

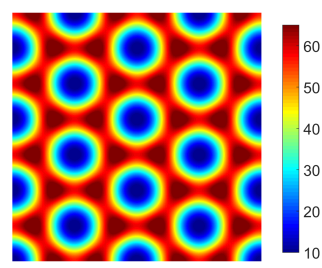

(d) $\mu=2.05, T=3000$

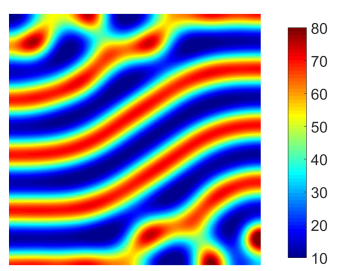

(f) $\mu=2.15, T=3000$

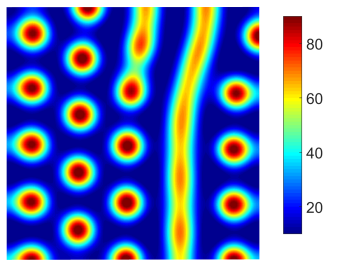

(h) $\mu=2.4, T=1000$

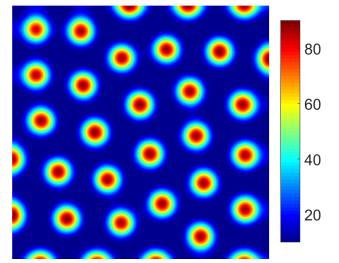

(j) $\mu=3, T=400$

Kansa method 2

Figure 3: Pattern transition in unit square domain with $d t=0.01, v=100.0, r=0.001, D_{1}=d D_{2}(d=0.0035)$, $D_{2}=0.27$ and $\mu \in\{2.04,2.05,2.15,2.4,3\}$ by the Kansa method under two initial conditions 


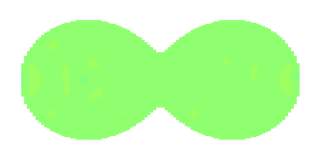

$\mu=2.04, T=400$

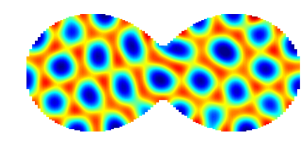

$\mu=2.05, T=3000$

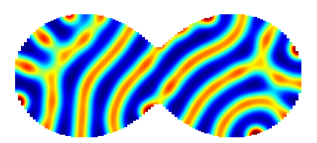

$\mu=2.15, T=3000$

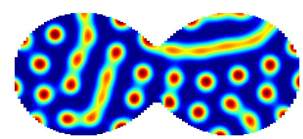

$\mu=2.4, T=3000$

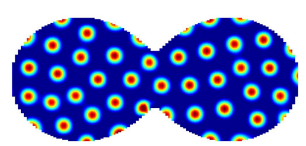

$$
\mu=3, T=400
$$

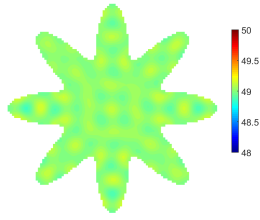

$\mu=2.04, T=10000$

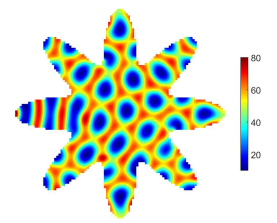

$\mu=2.05, T=5000$

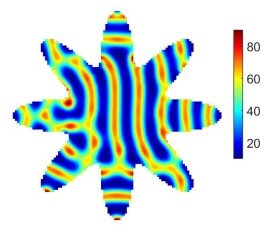

$\mu=2.15, T=3000$

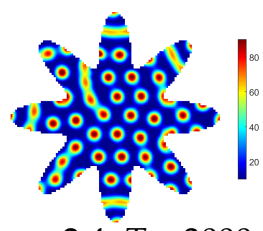

$\mu=2.4, T=3000$

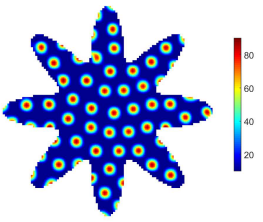

$\mu=3, T=400$

Figure 4: Pattern formations in $\Omega_{1}$ and $\Omega_{2}$ defined in Appendix when $\Delta t=0.01, v=100.0, r=0.001, D_{1}=d D_{2}$ $(d=0.0035), D_{2}=0.27$ and $\mu \in\{2.04,2.05,2.15,2.4,3\}$ with $N_{i}=884, N_{b}=222$ points for $\Omega_{1}$ and $N_{i}=884, N_{b}=969$ points for $\Omega_{2}$ 


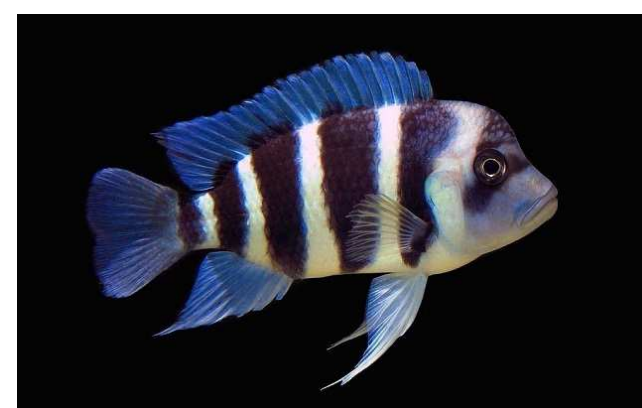

(a)

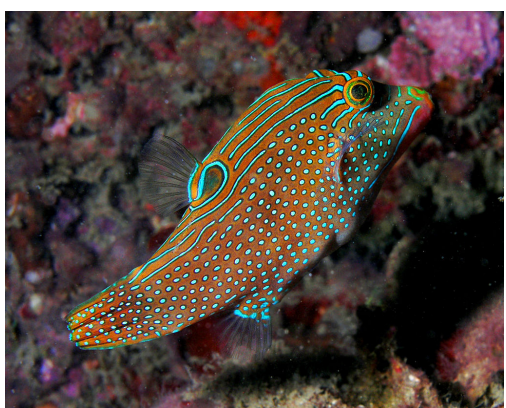

(b)

Figure 5: (a): frontosa fish [1] and (b): pufferfish [2].

\subsection{Mixed patterns of steady state, strips and spots}

Some real-life fishes have mixed patterns of steady state, spots and strips. From Fig. 5(a), the frontosa fish has vertical strips on its body and keep steady state on its tail. The sharpnose pufferfish possess both strips and spots on its body from Fig. 5(b). Before considering complicated fish-shape domains, we first try to generate similar patterns on $\Omega_{1}$ and $\Omega_{2}$. Mixed patterns can be generated by setting $\mu$ as the characteristic function

$$
\mu(x, y)= \begin{cases}\mu_{1} & \text { for }(x, y) \in P_{1}, \\ \mu_{2} & \text { for }(x, y) \in P_{2}: \Omega \backslash P_{1},\end{cases}
$$

where $\mu_{1}$ and $\mu_{2}$ are values corresponding to steady state, strip, or spot pattern from Section 4.2. When $\mu_{1}$ and $\mu_{2}$ takes values corresponding to steady state and strips, Figs. 6(a) and (c) show that expected mixed patterns from steady state to vertical strips are generated. If we set $\mu_{1}$ for spots and $\mu_{2}$ for strips, from Figs. $6(\mathrm{~b})$ and (d), we can have mixed patterns of horizontal strips and spots. We conclude that mixed patterns can be generated when $\mu$ takes as characteristic functions.

Next, we try to set $\mu$ as in Eq. (4.2) to generate patterns in frontosa fishes and sharpnose pufferfish in Fig. 5. Domains of these two fishes are constructed as $\Omega_{3}$ in Eqs. (A.3a)(A.3b) for frontosa fishes and $\Omega_{4}$ in Eq. (A.4a)-(A.4b) for sharpnose pufferfish as in the Appendix. Uniformly distributed $N_{i}=1743$ and $N_{b}=301$ discrete points are used in the domain and on the boundary of $\Omega_{3}$. Similar patterns with fronsta fishes are formed in Fig. 7(a) with $\mu_{1}$ and $\mu_{2}$ taking same values as in Fig. 6(a) and (c). Fig. 7(b) is the profile of the parameter $\mu$ in $\Omega_{3}$. For sharpnose pufferfishes, we use equally spaced trial centers $N_{i}=1746$ and $N_{b}=218$ in $\Omega_{4}$. Patterns of sharpnose pufferfish in Fig. 7(c) are formed by using same setting for $\mu_{1}$ and $\mu_{2}$ as in Figs. 6(b) and (d). Fig. 7(d) plot spatially changed $\mu$ used to generate the patterns.

Definitions of subdomain $P_{1}, P_{2}$ and specified values of $\mu_{1}, \mu_{2}$ for each Fig. in this part are shown in Table 3. 


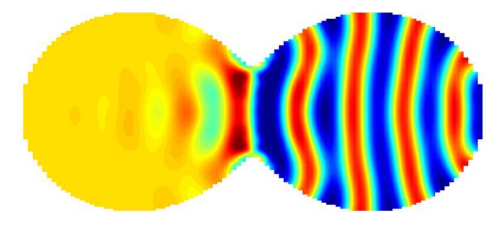

(a) $T=1000$

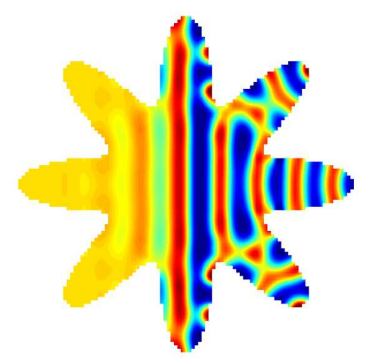

(c) $T=1000$

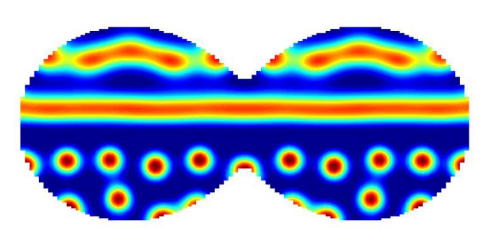

(b) $T=1000$

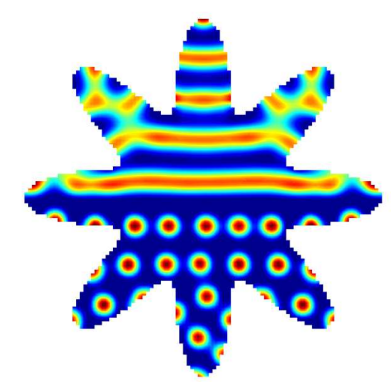

(d) $T=1000$

Figure 6: Pattern formations by $\Delta t=0.01, v=100.0, r=0.001, D_{1}=d D_{2}(d=0.0035), D_{2}=0.27$ and $\mu$ as the scharacteristic function in Eq. (4.2) with $\mu_{1}, \mu_{1}, P_{1}, P_{2}$ as in the Table 3.

\subsection{Patterns of varying size spots}

From Fig. 8(a), patterns of the stingary are spots with different size both on body and tail. In this part, we focus on construct this kind of patterns.

We first generate varying size spots on some simple domains. In [35], by the finite difference method, patterns with varying size spots can be formed by changing $\mu$ sinusoidally in the square domain as

$$
\mu(x, y)=p(1+0.5 \cos (q \pi x) \cos (q \pi y)) \quad \text { for }(x, y) \in \Omega,
$$

with $p$ and $q$ are two constants which can be used to adjust size of spots. Here, we use the same strategy to generate patterns on different domains. For domain $\Omega_{1}$ and $\Omega_{2}$, Fig. 9 shows our simulation results when $p=3$ and $q=3.5$ in Eq. (4.3). Patterns with two kinds different size spots are formed on both domains. It is once again observed that the domain shape has little influence to patterns.

We then apply $\mu$ in the Eq. (4.3) to construct patterns in a stingray-shape domain $\Omega_{5}$ defined in Eq. (A.5a)-(A.5b) from Appendix. We use $N_{i}=2155$ uniformly distributed points in the domain and $N_{b}=241$ discrete points on the boundary. Based on different 


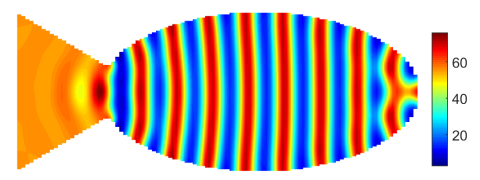

(a) $T=1000$

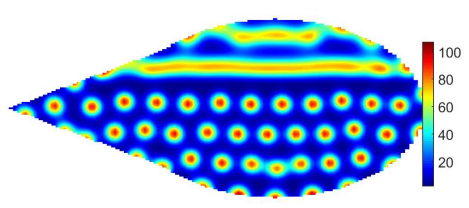

(c) $T=400$

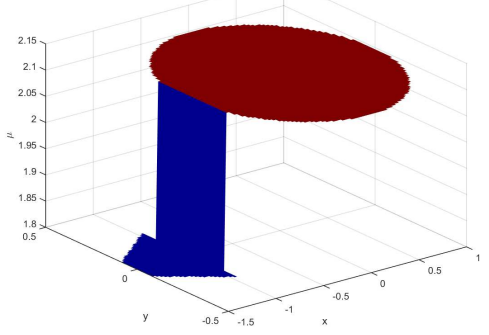

(b)

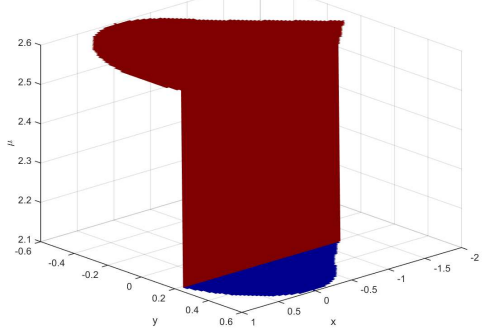

(d)

Figure 7: Pattern simulations of frontosa and sharpnose pufferfishes with $\Delta t=0.01, v=100.0, r=0.001, D_{1}=d D_{2}$ $(d=0.0035), D_{2}=0.27$ and $\mu$ as characteristic functions in Eq. (4.2) with $\mu_{1}, \mu_{2}, P_{1}, P_{2}$ as in the Table 3.

kinds spot patterns observed from stingray in Fig. 8(a), the domain $\Omega_{5}$ is divided into three patches

$$
P_{1}:\left\{\sqrt{x^{2}+y^{2}} \leq 0.6\right\}, \quad P_{2}: \Omega \cap\{x \leq-0.94\}, \quad P_{3}: \Omega_{5} \backslash\left(P_{1} \cup P_{2}\right) .
$$

We use different formulas of $\mu$ on $P_{1}, P_{2}$ and $P_{3}$ as

$$
\mu(x, y)= \begin{cases}2.5(1+0.5 \cos (3.5 \pi x) \cos (3.5 \pi y)) & \text { for }(x, y) \in P_{1} \\ 2.5(1+0.5 \cos (2.5 \pi x) \cos (2.5 \pi y)) & \text { for }(x, y) \in P_{2} \\ 3.0 & \text { for }(x, y) \in P_{3}\end{cases}
$$

Fig. 10(a) plot the pattern of stingray with final time $T=500$ and values of bifurcation parameter $\mu$ used in $\Omega_{5}$ is plotted in Fig. 10(b). It can be seen patterns of two different spots formed on $P_{1}$ and $P_{2}$. For subdomain $P_{3}$, smallest equally sized spots are generated since constant $\mu$ was used. 


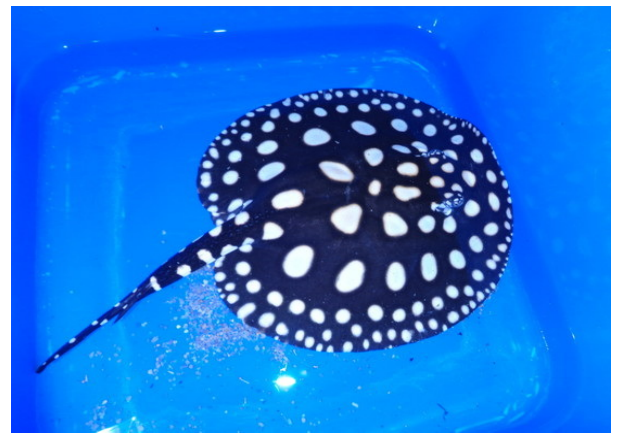

(a)

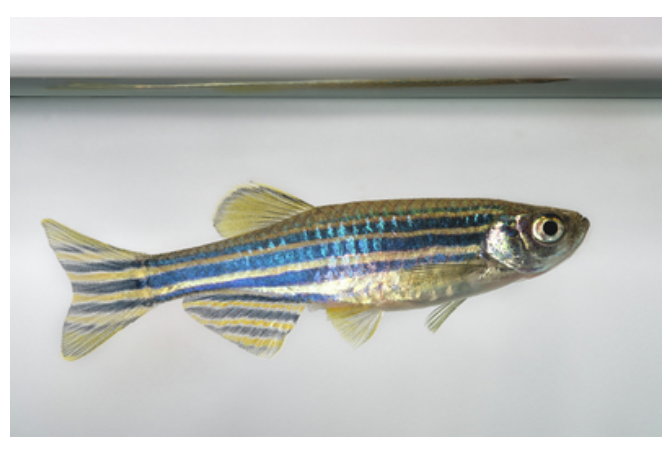

(b)

Figure 8: (a): stingray [3] and (b): zebrafish [4].
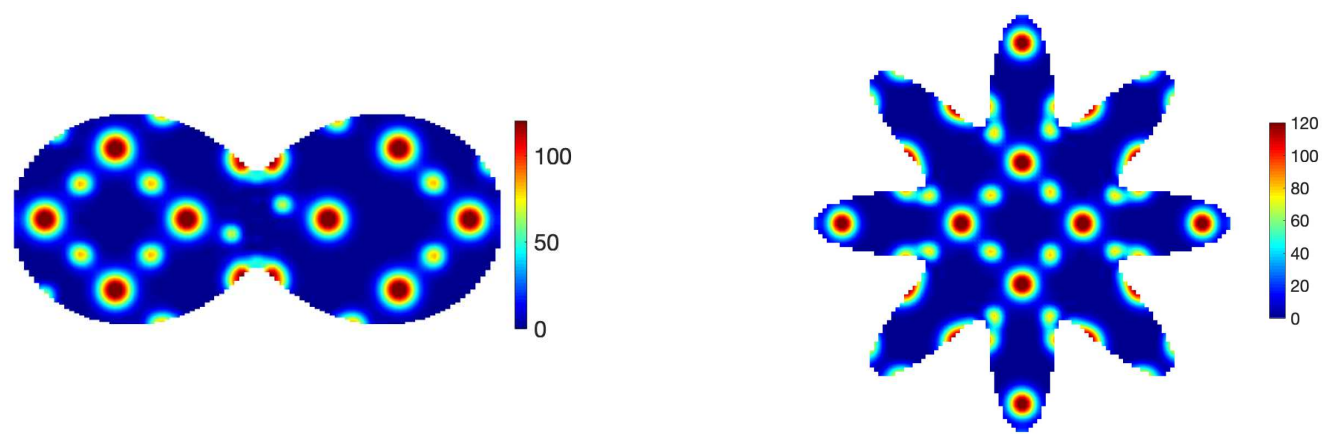

Figure 9: Pattern formation at $T=400$ when $d t=0.01, v=100.0, r=0.001, D_{1}=d D_{2}(d=0.0035), D_{2}=0.27$ and $\mu=3.0(1+0.5 \cos (3.5 \pi x) \cos (3.5 \pi y))$.

\subsection{Strips along fixed direction}

Based on findings in Section 4.2, strip patterns can be formed with $\mu \in[2.1,2.3]$. However, the direction of strips change with the initial conditions and cannot be controlled. Yet, some animal patterns in nature are going along some fixed directions. For example, patterns on zebrafishes are straight strips from head to tail in Fig. 8(b). In this part, we provide two methods to generate such patterns.

In the first case, straight strips are formed with a characteristic function $\mu$ as in Eq. (4.2) which we used to simulate patterns on frontosa fishes in Fig. 7. In order to obtain strips along horizontal direction, we take $\mu_{1}$ and $\mu_{2}$ with both corresponding to strips in Eq. (4.2). Figs. 11(a), (c) and (e) show resulting patterns. We can observe that strip patterns along the $x$ axis were formed in the square domain. Horizontal patterns were also formed in $\Omega_{1}$ and $\Omega_{2}$ although some irregular patterns near boundary appeared. To 


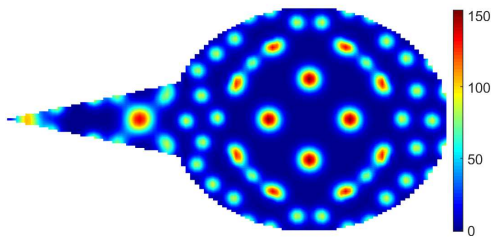

(a)

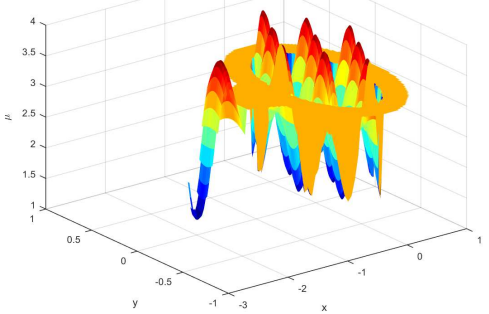

(b)

Figure 10: Pattern simulation of stingray at $T=500$ with $\Delta t=0.01, v=100.0, r=0.001, D_{1}=d D_{2}(d=0.0035)$, $D_{2}=0.27$ and the bifurcation parameter takes as $\mu=2.5(1+0.5 \cos (3.5 \pi x) \cos (3.5 \pi y))$ for $\sqrt{x^{2}+y^{2}}<0.6$, $\mu=2.5(1+0.5 \cos (2.5 \pi x) \cos (2.5 \pi y))$ for $x<-0.94$, and $\mu=3$ for elsewhere.

further construct patterns on zebrafishes, we apply $\mu$ as in Eq. (4.2) in $\Omega_{3}$ with values of $\mu_{i}, i=1,2$ corresponding to strips. Fig. 12(b) plots parameter setting in domain and the resulting simulation is shown in Fig. 12(a). Desired three horizonal strips are formed which is same as patterns on zebrafish in Fig. 8(b).

Numerically results show that strips along the $x$ axis can be formed when $\mu$ changes linearly along the $y$ axis from $\mu_{1}$ to $\mu_{2}$ with both values corresponding to strip patterns. However, strips are not straight for the influence of domain shapes. Therefore, in our second method, we divide the domain into two patches and apply constant and linearly changing $\mu$ in these two patches respectively as

$$
\mu= \begin{cases}\mu_{1}+\frac{\mu_{2}-\mu_{1}}{y_{2}-y_{1}}\left(y-y_{1}\right) & \text { for }(x, y) \in P_{1}, \\ \mu_{2} & \text { for }(x, y) \in P_{2} .\end{cases}
$$

When we use $\mu_{1}$ and $\mu_{2}$ in Eq. (4.5) both corresponds to strips, Figs. 11(b), (d) and (f) show simulations in the square domain, $\Omega_{1}$ and $\Omega_{2}$. It can be seen straight strip patterns along the $x$-axis were formed in most region of the domain except small perturbation near boundary of $\Omega_{2}$ and $\Omega_{3}$. With same values $\mu_{1}$ and $\mu_{2}$ used in different patches of $\Omega_{3}$ as in Fig. 12(d), we can construct strips in the Fig. 12(c) which is similar with patterns on zebrafishes.

Definitions of sub-domains $P_{1}, P_{2}$ and specific values of parameters in Eq. (4.2) and Eq. (4.5) for construct patterns in this part are shown in Table 4.

\subsection{Circular and semicircular patterns}

In this part, we aim to construct circular strips centered with an circle on mimic octopus in Fig. 13(b) and semicircular strips centered with an ellipse on angelfish in Fig. 13(a). 


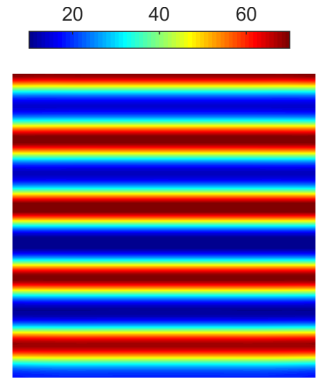

(a)

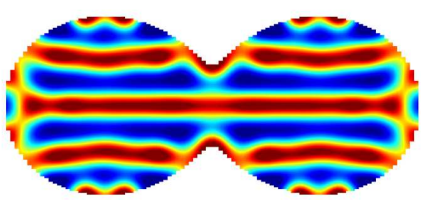

(c)

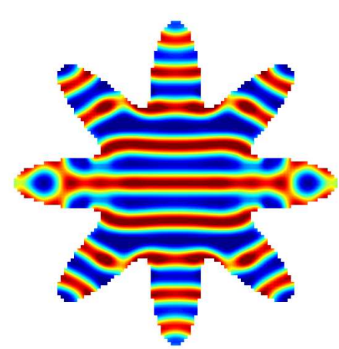

(e)

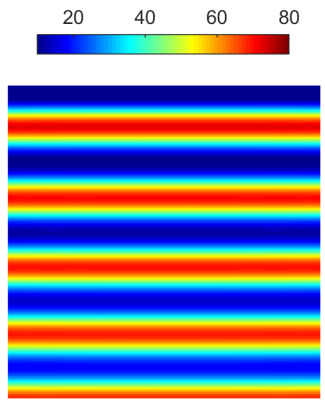

(b)

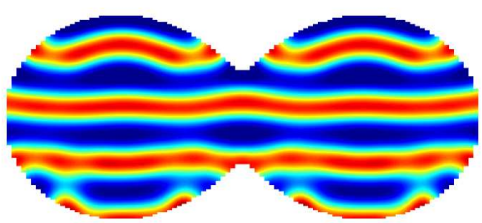

(d)

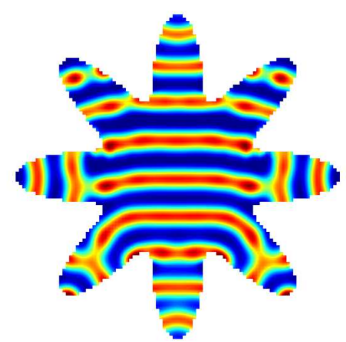

(f)

$T=1000$

Figure 11: Pattern formations with $\Delta t=0.01, v=100.0, r=0.001, D_{1}=d D_{2}(d=0.0035), D_{2}=0.27$ and spatially changed parameters $\mu$ as step functions in Eq. (4.2) and function as in Eq. (4.5) with parameters values shown in Table 4.

In [35], when the domain are divided into two patches with a small rectangle as boundary, circular strips can be generated by finite difference method. Motivated by this, we use a similar strategy to built circular and semicircular strips. Instead of using rectangles as boundaries of two patches, we use a small ellipse and spatially changed 


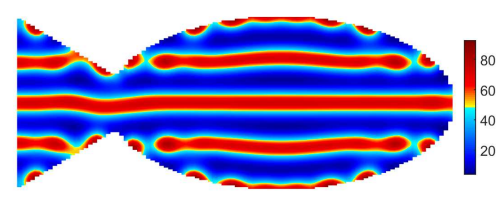

(a) $T=500$

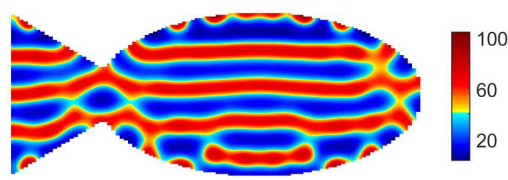

(c)

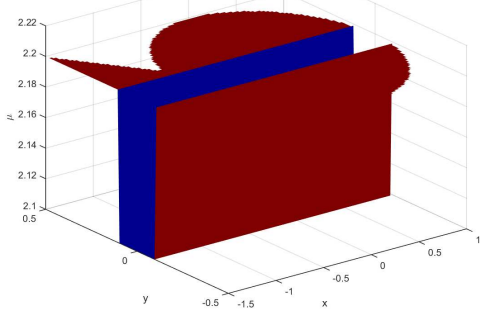

(b)

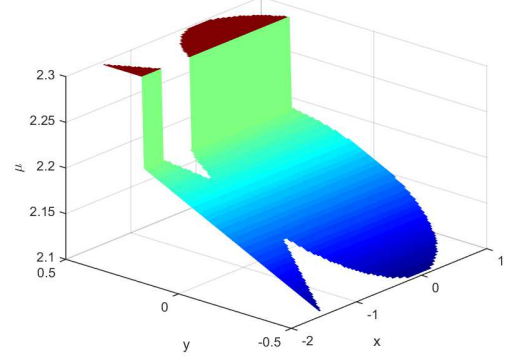

(d) $T=1000$

Figure 12: Pattern simulations of zebrafishes with $N_{i}=1743, N Z_{b}=276, \Delta t=0.01, v=100.0, r=0.001, D_{1}=d D_{2}$ $(d=0.0035), D_{2}=0.27$ and the bifurcation parameter takes as : (a) step functions Eq. (4.2), (c) function as in Eq. (4.5) with parameters values as in Table 4.

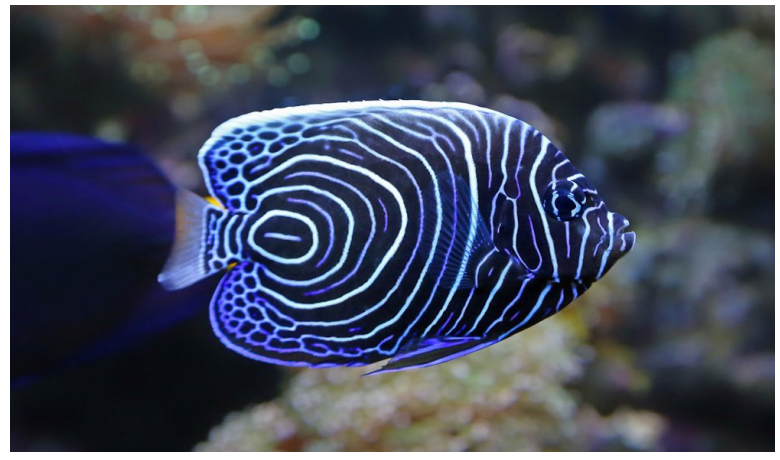

(a)

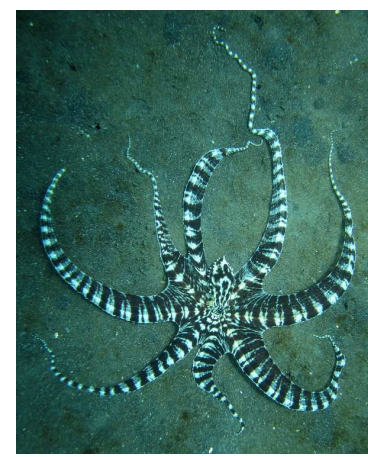

(b)

Figure 13: (a): angelfish [5] and (b): mimic octopus [6].

parameter $\mu$ set as

$$
\mu(x, y)= \begin{cases}\mu_{1} & \text { for } P_{1}=:\left\{(x, y) \mid\left(\frac{x-x_{c}}{a}\right)^{2}+\left(\frac{y-y_{c}}{b}\right)^{2} \leq 1,(x, y) \in \Omega\right\}, \\ \mu_{2} & \text { for }(x, y) \in P_{2}: \Omega \backslash P_{1}\end{cases}
$$




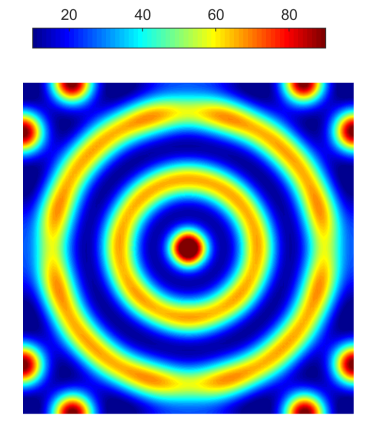

(a)

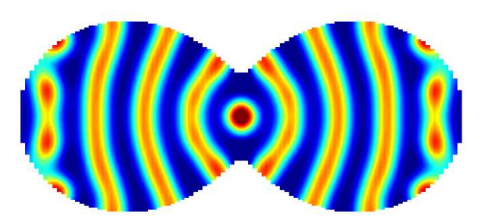

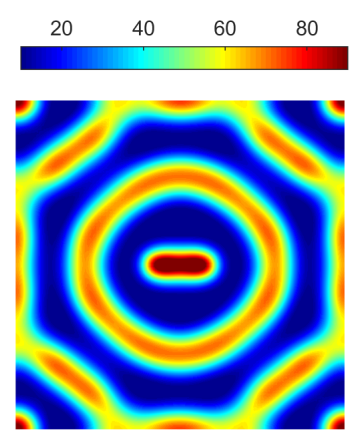

(b)

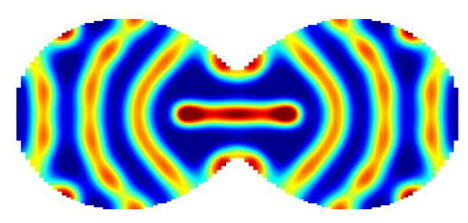

(d)

Figure 14: Patterns with $\Delta t=0.01, v=100.0, r=0.001, D_{1}=d D_{2}(d=0.0035), D_{2}=0.27$ and $\mu$ as in Eq. (4.6) with unknown parameters in Table 5.

When the values of $a$ and $b$ are equal in $P_{1}$ of Eq. (4.6), boundaries of these two patches become a circle. We first consider this case. Circular and semicircular strips centered with a spot can be formed if $\mu_{1}$ and $\mu_{2}$ take values corresponding to steady state and strips. Fig. 14(a) shows that circular strips with spots in the center and near boundary are formed in the unit square domain. Semicircular strips centered with a spot formed in Fig. 14(c) for $\Omega_{1}$. When applying similar setting of $\mu$ to $\Omega_{2}$, Fig. 15(a) shows that strips on eight arms and circular strips centered with a spot on bod are formed, which is similar to patterns on mimic octopus in Fig. 13(b). Fig. 15(b) plot spatially changed $\mu$ used in this simulation.

Lastly, we consider patterns by using an ellipse as boundary of two patches. Values of $\mu_{1}$ for steady state and $\mu_{2}$ for strips are also used in this case. From Fig. 14(b) and (d), circular strips centered at an ellipse are formed for the square domain and semicircular strips centered with an ellipse formed for $\Omega_{2}$, respectively. We use this setting of $\mu$ to 


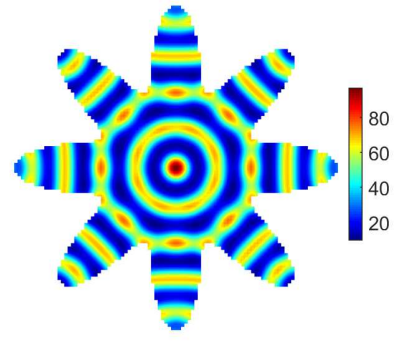

(a) $T=500$

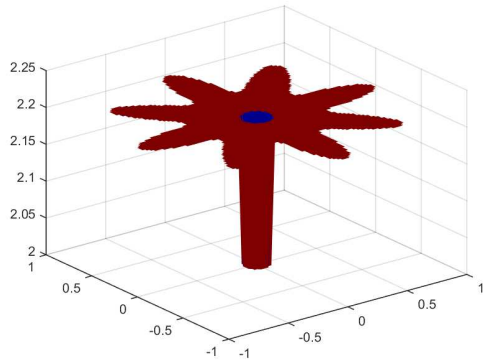

(b)

Figure 15: Simulations of patterns on minic octopus when $N_{i}=969, N_{b}=260, \Delta t=0.01, v=100.0, r=0.001, D_{1}=$ $d D_{2}(d=0.0035), D_{2}=0.27, \mu=2.0$ for $\sqrt{x^{2}+y^{2}}<0.1$ and $\mu=2.2$ elsewhere.

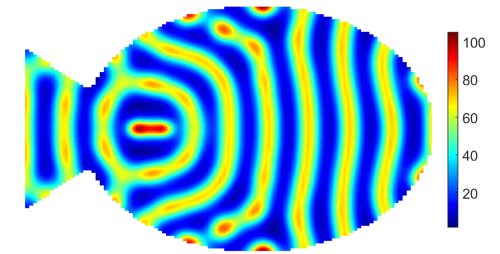

(a) $T=1000$

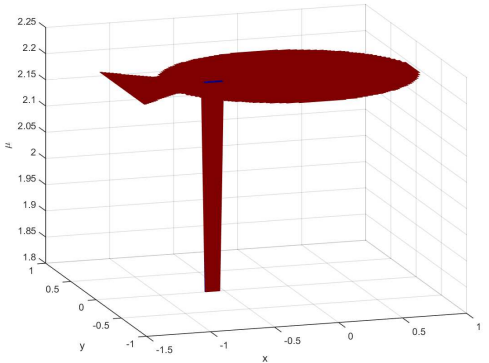

(b)

Figure 16: Pattern simulations of angelfishes with $N_{i}=1715, N_{b}=207, \Delta t=0.01, v=100.0, r=0.001, D_{1}=$ $d D_{2}(d=0.0035), D_{2}=0.27, \mu=1.8$ for $\left(\frac{x+0.6}{0.1}\right)^{2}+\left(\frac{y}{0.01}\right)^{2}<1$ and $\mu=2.2$ elsewhere.

construct patterns on angelfishes. The domain $\Omega_{6}$ defined in Eqs. (A.6a)-(A.6b) in Appendix. Uniformly distributed discrete sets with $N_{i}=1715$ and $N_{b}=207$ in the domain and on boundary are used. When we use parameter $\mu$ in Fig. 16(b), semicircular strips centered at a small ellipse close to tail are formed in Fig. 16(a) which is similar to patterns on angelfishes (Fig. 13(a)).

Specific values of $x_{c}, y_{c}, a$ and $b$ in Eq. (4.6) used for simulations desired patterns in this part are shown in Table 5.

\section{Conclusions}

We studied Turing instability of Gierer-Meinhardt activator-inhibitor models by a linear analysis and evaluate the critical value of bifurcation parameter analytically. Combining 
the SBDF2 method for time discretization and the meshless Kansa method for spatial discretization, pattern transition were numerically simulated on irregular domains. We also construct complex patterns appeared in nature. Varying size spots patterns can be formed by changed parameter sinusoidally. Mixed patterns of steady state, strips or spots are formed by some characteristic functions of bifurcation parameter on different patches of domain. Circular patterns can be built by the step function of parameter with boundaries of different patches set as circles or ellipses. We also constructed different animal shape domains and simulated patterns appeared in reality.

\section{Appendix}

\section{A.1 Definitions of irregular domains}

Beside the unit square domain, peanut shape domain, asterisk domain and some animal shape domains also used in our numerical simulation. We state definitions of them here.

1. The peanut shape domain defined as

$$
\Omega_{1}=:\left\{(r, \theta) \mid r=\frac{2}{5}\left(\frac{3}{2}+\cos (2 \theta)\right), \theta \in[0,2 \pi]\right\} .
$$

2. The asterisk shape domain defined as

$$
\Omega_{2}=:\left\{(r, \theta) \mid r=\frac{2}{5}\left(\frac{3}{2}+\cos (4 \theta)^{2}\right), \theta \in[0,2 \pi]\right\} .
$$

3. Domain $\Omega_{3}=: \Omega_{3}^{1} \cup \Omega_{3}^{2}$ to simulate patterns on frontosa fish and zebrafishes is constructed as

$$
\begin{aligned}
& \Omega_{3}^{1}=\{(x, y) \mid-0.5+0.61(x+1.5) \leq y \leq 0.5-0.61(x+1.5), x \in[-1.5,-0.94]\}, \\
& \Omega_{3}^{2}=\left\{(x, y) \mid x^{2}+\frac{y^{2}}{0.5^{2}}=1, x \in[-0.94,1], y \in[0.5,5]\right\} .
\end{aligned}
$$

4. For sharpnose pufferfish, triangles and ellipse are used to built domain $\Omega_{4}=: \Omega_{4}^{1} \cup$ $\Omega_{4}^{2}$ as

$$
\begin{aligned}
& \Omega_{4}^{1}=\left\{(x, y) \mid x^{2}+\frac{y^{2}}{0.6^{2}}=1, x \in[-0.74,1], y \in[-0.6,6]\right\}, \\
& \Omega_{4}^{2}=\{(x, y) \mid-0.37(x+1.8) \leq y \leq 0.37(x+1.8), x \in[-1.8,-0.74]\} .
\end{aligned}
$$


5. Domain $\Omega_{5}=: \Omega_{5}^{1} \cup \Omega_{5}^{2}$ used to simulate patterns on stingray in Fig. 8 is constructed as

$$
\begin{aligned}
& \Omega_{5}^{1}=\left\{(x, y) \mid x^{2}+\frac{y^{2}}{0.8^{2}}=1, x \in[-0.94,1], y \in[-0.8,8]\right\}, \\
& \Omega_{5}^{2}=\{(x, y) \mid-0.21(x+2.2) \leq y \leq 0.21(x+2.2), x \in[-2.2,-0.94]\} .
\end{aligned}
$$

6. Domain $\Omega_{6}=: \Omega_{6}^{1} \cup \Omega_{6}^{2}$ used to simulate patterns on anglefishes is defined as

$$
\begin{aligned}
& \Omega_{6}^{1}=\left\{(x, y) \mid x^{2}+\frac{y^{2}}{0.7^{2}}=1, x \in[-0.94,1], y \in[-0.7,0.7]\right\}, \\
& \Omega_{6}^{2}=\{(x, y) \mid 0.47-0.65(x+1.3) \leq y \leq 0.47+0.95(x+1.3), x \in[-1.3,-0.94]\} .
\end{aligned}
$$

\section{A.2 Parameter settings}

In order to simulate patterns appeared in nature, we provide different settings for spatially changed bifurcation parameters, such as characteristic functions or trigonometric functions in Section 4. In some cases, we also need to divided domains into different patches and used different values of $\mu$ on each patch. In this part, we show specific values of parameters and definitions of different patches of domains used in numerical simulations. Table 3 is parameter values used for mixed patterns of steady state, strips and spots in Section 4.3. Table 4 is parameter values used for strips along fixed direction in Section 4.5. Table 5 is parameter values used for circular and semicircular strips in Section 4.6.

Table 3: Parameter values for mixed patterns of steady state, strips and spots in Section 4.3.

\begin{tabular}{||c|c|c|c|c||}
\hline Fig. & $\mu_{1}$ & $\mu_{2}$ & $P_{1}$ & $P_{2}$ \\
\hline Figs. 6(a), (c) & 1.8 & 2.1 & $\Omega_{i} \cap\{x \leq 0\}, i=\{1,2\}$ & $\Omega_{i} / P_{1}$ \\
\hline Figs. 6(b), (d) & 2.6 & 2.1 & $\Omega_{i} \cap\{y \leq 0\}, i=\{1,2\}$ & $\Omega_{i} / P_{1}$ \\
\hline Fig. 7(a) & 1.8 & 2.1 & $\Omega_{3}^{1}$ in Eq. (A.3a) & $\Omega_{3}^{2}$ in Eq. (A.3b) \\
\hline Fig. 7(c) & 2.6 & 2.1 & $\Omega_{4} \cap\{y \leq 0.2\}$ & $\Omega_{4} / P_{1}$ \\
\hline
\end{tabular}

Table 4: Parameter values for strips along fixed direction in Section 4.5.

\begin{tabular}{||c|c|c|c|c|c|c||}
\hline Fig. & $\mu_{1}$ & $\mu_{2}$ & $P_{1}$ & $P_{2}$ & $y_{1}$ & $y_{2}$ \\
\hline Fig. 11(a) & 2.1 & 2.2 & {$[0,1] \times[0.5,1]$} & {$[0,1] \times[0,0.5]$} & & \\
\hline Fig. 11(b) & 2.1 & 2.3 & {$[0,1] \times[0,0.9]$} & {$[0,1] \times[0.9,1]$} & 0 & 0.9 \\
\hline Figs. 11(c), (e) & 2.1 & 2.2 & $\begin{array}{c}\Omega_{i} \cap\{|y| \leq 0.3\}, \\
i=\{1,2\}\end{array}$ & $\Omega_{i} \backslash P_{1}$ & & \\
\hline Fig. 11(d) & 2.1 & 2.3 & $\Omega_{1} \cap\{y \leq 0.35\}$ & $\Omega_{1} \backslash P_{1}$ & -0.5 & 0.35 \\
\hline Fig. 11(f) & 2.1 & 2.3 & $\Omega_{2} \cap\{y \leq 0.3\}$ & $\Omega_{2} \backslash P_{1}$ & -1 & 0.3 \\
\hline Fig. 12(a) & 2.1 & 2.2 & $\Omega_{3} \cap\{y \leq 0.3\}$ & $\Omega_{3} \backslash P_{1}$ & & \\
\hline Fig. 12(c) & 2.1 & 2.3 & $\Omega_{3} \cap\{y \leq 0.3\}$ & $\Omega_{3} \backslash P_{1}$ & -0.5 & 0.3 \\
\hline
\end{tabular}


Table 5: Parameter values for circular and semicircular strips in Section 4.6.

\begin{tabular}{||c|c|c|c|c|c|c||}
\hline Fig. & $a$ & $b$ & $x_{c}$ & $y_{c}$ & $\mu_{1}$ & $\mu_{2}$ \\
\hline Fig. 14(a) & 0.05 & 0.05 & 0.5 & 0.5 & 2.0 & 2.2 \\
\hline Fig. 14(b) & 0.12 & 0.02 & 0.5 & 0.5 & 1.8 & 2.2 \\
\hline Fig. 14(c) & 0.07 & 0.07 & 0 & 0 & 2.0 & 2.3 \\
\hline Fig. 14(d) & 0.3 & 0.05 & 0 & 0 & 1.8 & 2.2 \\
\hline Fig. 15(a) & 0.1 & 0.1 & 0 & 0 & 2.0 & 2.2 \\
\hline Fig. 16(a) & 0.1 & 0.01 & -0.6 & 0 & 1.8 & 2.2 \\
\hline
\end{tabular}

\section{Acknowledgements}

This work was partially supported by a Hong Kong Research Grant Council GRF Grant, and a Hong Kong Baptist University FRG Grant.

\section{References}

[1] https://www . pinterest.com/pin/521010250618931794/.

[2] https://en.wikipedia.org/wiki/Canthigaster_solandri.

[3] https://www.numa.sk/en/product/leopoldi-stingray.

[4] https://www.skretting.com/en-US/species/zebrafish/.

[5] https://www.dreamstime.com/stock-photos-close-up-view-juvenile-emperor -angelfish-pomacanthus-imperator-image40404793.

[6] https://en.wikipedia.org/wiki/Mimic_octopus .

[7] R. F. AlLEN, Turing instabilities and spatial pattern formation in one-dimension, (2003).

[8] R. A. Barrio, C. Varea, J. L. Aragón, And P. K. Maini, A two-dimensional numerical study of spatial pattern formation in interacting turing systems, Bulletin of Mathematical Biology, 61(3) (1999), pp. 483-505.

[9] M. BAURMANN, T. GROSS, AND U. FEUDEL, Instabilities in spatially extended predator-prey systems: spatio-temporal patterns in the neighborhood of turing-hopf bifurcations, Journal of Theoretical Biology, 245(2) (2007), pp. 220-229.

[10] B. BunOW, J. P. KerneveZ, G. JOLy, AND D. THOMAS, Pattern formation by reaction-diffusion instabilities: Application to morphogenesis in drosophila, Journal of Theoretical Biology, 84(4) (1980), pp. 629-649.

[11] K. C. CHEUnG AND L. LING, A kernel-based embedding method and convergence analysis for surfaces PDEs, SIAM J. Sci. Comput., 40(1) (2018), pp. A266-A287.

[12] K. C. CHEUNG, L. LING, AND R. SCHABACK, $H^{2}$-convergence of least-squares kernel collocation methods, SIAM Journal on Numerical Analysis, 56(1) (2018), pp. 614-633.

[13] M. DEHGHAN AND M. ABBASZADEH, Numerical study of three-dimensional turing patterns using a meshless method based on moving kriging element free galerkin (efg) approach, Computers and Mathematics with Applications, 72(3) (2016), pp. 427-454.

[14] M. Dehghan, M. AbBaszadeh, And A. MOHEBBi, The use of element free galerkin method based on moving kriging and radial point interpolation techniques for solving some types of turing models, Engineering Analysis with Boundary Elements, 62 (2016), pp. 93-111. 
[15] R. I. FERNANDES AND G. FAIRWEATHER, An adi extrapolated crank-nicolson orthogonal spline collocation method for nonlinear reaction-diffusion systems, Journal of Computational Physics, 231(19) (2012), pp. 6248-6267.

[16] S. C FERREIRA JR., M. L. MARTINS, AND M. J. Vilela, Reaction-diffusion model for the growth of avascular tumor, Physical Review E, 65(2) (2002), 021907.

[17] Z. Y. XING, AND L. P. WEN, The fast implementation of the ADI-CN method for a class of two dimensional Riesz space-fractional diffusion equations, Adv. Appl. Math. Mech., 11(4) (2019), pp. 942-956.

[18] L. P. CHEN, AND Y. P. CHEN, A novel discretization method for semilinear reaction-diffusion equation, Adv. Appl. Math. Mech., 10(2) (2018) pp. 409-423.

[19] R. FitzHuGH, Mathematical models of threshold phenomena in the nerve membrane, The Bulletin of Mathematical Biophysics, 17(4) (1955), pp. 257-278.

[20] A. Gierer AND H. MeINHARDT, A theory of biological pattern formation, Kybernetik, 12(1) (1972), pp. 30-39.

[21] Y. C. HON AND R. SCHABACK, On unsymmetric collocation by radial basis functions, Applied Mathematics and Computation, 119(2-3) (2001), pp. 177-186.

[22] Z. JANNESARI AND M. TATARI, A meshfree technique for numerical simulation of reactiondiffusion systems in developmental biology, Advances in Applied Mathematics and Mechanics, 9(5) (2017), pp. 1225-1249.

[23] M. KAMRANIAN AND M. DEHGHAN, The finite point method for reaction-diffusion systems in developmental biology, CMES Comput. Model. Eng. Sci., 82(1) (2011), pp. 1-27.

[24] E. J. KANSA, Multiquadrics-a scattered data approximation scheme with applications to computational fluid-dynamics. I. Surface approximations and partial derivative estimates, Computers and Mathematics with Applications, 19(8-9) (1990), pp. 127-145.

[25] E. J. KANSA, Multiquadrics-a scattered data approximation scheme with applications to computational fluid-dynamics. II. Solutions to parabolic, hyperbolic and elliptic partial differential equations, Computers and Mathematics with Applications, 19(8-9) (1990), pp. 147-161.

[26] L. LING, R. OPFER, AND R. SCHABACK, Results on meshless collocation techniques, Engineering Analysis with Boundary Elements, 30(4) (2006), pp. 247-253.

[27] L. LING AND R. SCHABACK, Stable and convergent unsymmetric meshless collocation methods, SIAM Journal on Numerical Analysis, 46(3) (2008), pp. 1097-1115.

[28] A. Madzvamuse, P. K. MAini, And A. J. WAthen, A moving grid finite element method for the simulation of pattern generation by turing models on growing domains, Journal of Scientific Computing, 24(2) (2005), pp. 247-262.

[29] M. MOHAmmadi, R. MOKHTARi, AND R. SCHABACK, A meshless method for solving the 2d brusselator reaction-diffusion system, Comput. Model. Eng. Sci., 101 (2014), pp. 113-138.

[30] J. D. MURRAY, Parameter space for turing instability in reaction diffusion mechanisms: a comparison of models, Journal of Theoretical Biology, 98(1) (1982), pp. 143-163.

[31] G. CATANIA, AND A. ZANARINI, Flexible multibody system dynamics by means of a spectralbased meshless approach, Adv. Appl. Math. Mech., 11(4) (2019) pp. 757-806.

[32] S. Y. YI, AND H. G. SUN, A hybrided trapezoidal-difference scheme for nonlinear time fractional fourth-order advection-dispersion equation based on chebyshev spectral collocation method, Adv. Appl. Math. Mech., 11(1) (2019), pp. 197-215.

[33] H. G. Othmer, K. PAinter, D. Umulis, And C. Xue, The intersection of theory and application in elucidating pattern formation in developmental biology, Mathematical Modelling of Natural Phenomena, 4(4) (2009), pp. 3-82.

[34] K. PAge, P. K. Maini, AND N. A. M. MonK, Pattern formation in spatially heterogeneous 
turing reaction-diffusion models, Physica D: Nonlinear Phenomena, 181(1-2) (2003), pp. 80101.

[35] K. M. Page, P. K. MaInI, AND N. A. M. MonK, Complex pattern formation in reactiondiffusion systems with spatially varying parameters, Physica D: Nonlinear Phenomena, 202(1-2) (2005), pp. 95-115.

[36] K. J. PAINTER, Models for pigment pattern formation in the skin of fishes, Mathematical Models for Biological Pattern Formation, pp. 59-81, Springer, 2001.

[37] S. J. RUUTH, Implicit-explicit methods for reaction-diffusion problems in pattern formation, Journal of Mathematical Biology, 34(2) (1995), pp. 148-176.

[38] S. A. SARRA, A local radial basis function method for advection-diffusion-reaction equations on complexly shaped domains, Applied Mathematics and Computation, 218(19) (2012), pp. 98539865.

[39] J. SCHNAKENBERG, Simple chemical reaction systems with limit cycle behaviour, Journal of Theoretical Biology, 81(3) (1979), pp. 389-400.

[40] A. M. TURING, The chemical basis of morphogenesis, Philosophical Transactions of the Royal Society of London, Series B, Biological Sciences, 237(641) (1952), pp. 37-72.

[41] C. C. XIE AND X. L. HU, Finite element simulations with adaptively moving mesh for the reaction diffusion system, Numerical Mathematics: Theory, Methods and Applications, 9(4) (2016), pp. 686-704. 